\title{
Bajtín y Benjamin: lecturas desde otros cronotopos (en torno a Goethe y otros temas)
}

\author{
Tatiana Bubnova
}

En este artículo se lleva a cabo una lectura paralela de algunos textos de dos pensadores del siglo xx: el ruso Mijaíl Bajtín (1895-1975) y el alemán Walter Benjamin (1892-1940). Realizada con una intención heurística, tal lectura está condicionada, como cualquier otra, por la circunstancia histórica, geográfica, social del lector, y por tal circunstancia se manifiesta como necesaria. Tal vez revele más sobre el lector que sobre el objeto; aun así, se realiza con la herramienta analítica proporcionada sobre todo por Bajtín y está provocada por la heterodoxia benjaminiana vista asimismo desde los conceptos del ruso. De igual manera, la lectura obedece a un malestar provocado por una inconsistencia ética con que estos productos intelectuales se han manipulado en el mercado académico.

Palabras clave: Bajtín, Benjamin, Goethe, Las afinidades electivas.

This paper proposes a parallel reading of some texts by Mijail Bajtin (18951975) and Walter Benjamin (1892-1940), two of the most influential critics of the $20^{\text {th }}$ Century. Although its heuristic approach is conditioned - as any other- by the social, historical and geographical circumstances of the reader, and that it might reveal more about the reader than about its object, this approach uses the analytic tools provided by Batjin and is induced by Benjamin's heterodoxy. This text is motivated as well by the uneasiness caused by the unethical way in which this intellectual works have been manipulated in the academic world. 

Tatiana Bubnova

Instituto de Investigaciones Filológicas, UNAM

\section{Bajtín y Benjamin: lecturas desde otros cronotopos (en torno a Goethe y otros temas)}

Se lleva el viento a la semilla de oro: ella se ha perdido, no volverá jamás.

Mandelstam

No existe nada muerto de una manera absoluta: cada sentido tendrá su fiesta de renacimiento.

BAJTín

Los epígrafes se contradicen. Si se toma en cuenta el hecho de que las ideas de Bajtín acerca de la comunicación verbal son autorreferentes, ${ }^{1}$ en la contradicción se puede encontrar un determinado sentido. En primer lugar, siguiendo a Mandelstam, resulta que, en efecto, lo que se pierde, se pierde para siempre, y es imposible recuperar el pasado en su plenitud. Pero, por otro lado, preferimos creer que "los manuscritos no $\operatorname{arden}^{2}, \mathrm{y}$

1 "Los textos de Bajtín son textos que se analizan a sí mismos, se trata de una filosofía del lenguaje que se ilustra a sí misma y está orientada al habla indirecta" (L. Gogotishvili y Gurevich, Baxtin kak filosof, 144; todas las traducciones del ruso son mías, salvo indicación de lo contrario).

${ }^{2}$ Como pretende Woland, o sea, el Satán, en el Maestro y Margarita de Bulgakov. 
ninguna palabra se muere completamente. La palabra sobrevive siempre, la cuestión es, ¿en qué forma? Es lo que deberían preguntarse a sí mismos todos aquellos que pretenden una vez más "haber comprendido" a Bajtín, tanto en el sentido positivo como negativo. Lo que no suele practicarse es la aplicación de la concepción bajtiniana acerca de la dialogicidad interna de la palabra al mismo proceso de recepción (occidental) de sus ideas, desde el mismo principio (hacia 1966) ${ }^{3}$ hasta el mero presente. A mi modo de ver, al hacerlo, daríamos con el "diálogo en el gran tiempo" que la palabra establece con los interlocutores pasados y futuros por una parte, y con la existencia histórica del enunciado bien arraigado en el presente por la otra, conceptos que aparecen como dos polos de su pensamiento que se condicionan mutuamente. Porque un enunciado históricamente concreto puede ser recibido, en el "gran tiempo", de una manera insospechada incluso para su propio autor, debido no sólo al contexto, sino también al potencial semántico de una palabra "neutral" (cfr. Voloshinov, El marxismo: la palabra no pertenece a nadie, por eso se "apropian" de ella los diversos grupos sociales, dándole connotaciones ideológicas encontradas). Esto es de sentido común. Pero, por extraño que parezca, en sus textos pueden encontrarse aspectos que no cuadran bien con su aparentemente "racional" y actualizada interpretación del lenguaje ligada a su enunciación instantánea, interpretación que nos resulta más cómoda cuando situamos el pensamiento bajtiniano en la esfera de una "prosaica". ${ }^{4}$ Por ejemplo, cuando Bajtín menciona el "ser expresivo y hablante", cuando habla del "ser que se revela a sí mismo" (Sobranie Sochinenii, tomos

${ }^{3}$ Cuando Kristeva presenta su informe, "Bakhtine, le mot, le dialogue, le roman" (publicado en Critique) en el seminario de Barthes.

${ }^{4}$ Cfr. la interpretación magistral y comúnmente aceptada del pensamiento bajtiniano como una "prosaica" (contraparte de la "poética"); es decir, en el sentido de empirismo y pragmatismo, en G. S. Morson y C. Emerson, Mikhail Bakhtin, Creation of a Prosaics. 
5 y 2,8 ), su postura hace contrapunto con las ideas de Benjamin, para quien el mundo y sus objetos anteriores a la "Caída" solían hablar en su propio lenguaje, el de su ser, que el hombre conoció mediante los nombres que les asignara de acuerdo con la tarea que Dios le había dado. Pero el lenguaje como conocimiento fue justamente la manifestación de la Caída. La función comunicativa del lenguaje resulta, de este modo, para Benjamin, una "función burguesa". ${ }^{5}$ De acuerdo con Bajtín, la naturaleza observa las acciones humanas en calidad de "testigo y juez" (Estética de la creación verbal, 341). La propia idea de la "Caída del lenguaje" hace, en mi opinión, un contrapunto con las reflexiones finales de Voloshinov, en El marxismo y la filosofía del lenguaje, acerca de la "palabra ideológica" que se vuelve irresponsable por apoyarse demasiado en la "palabra ajena" para justificar la posición propia (Voloshinov, El marxismo, 173): la caída en el registro de la "prosaica". Mientras tanto, Benjamin habla acerca de un utópico "lenguaje puro" ("La tarea del traductor", artículo publicado en 1923), para el cual la "falsa conciencia" es imposible.

No obstante, existen otros temas que invitan a colocar a ambos pensadores en planos paralelos. Así, el "marxismo gótico"6 de Benjamin, en la perspectiva del cual el surrealismo, si bien observado desde una distancia crítica, no dejaba de ser una fuente de la "iluminación mundana del pensamiento" (Benja-

${ }^{5}$ Cfr. Benjamin, Obras, II 1, 144-161 ("Sobre el lenguaje en cuanto tal y sobre el lenguaje del hombre").

${ }^{6}$ Es la opinión de M. Löwy. No hay que perder de vista que el marxismo de Benjamin se caracteriza, además, por su pesimismo activo y productivo, “' organizado', práctico, orientado plenamente al propósito de impedir, mediante todos los modos posibles, la llegada de lo peor" (Löwy, "Walter Benjamin y el surrealismo", 86; como se puede ver, Löwy le atribuye a Benjamin cualidades de profeta) que estaba entonces a punto de llegar, frente a la fe irresponsable y cándida en el esquema lineal y optimista del progreso que solían profesar los partidos burgueses y socialdemócratas. Conviene agregar que el marxismo-leninismo soviético compartía esta idea simplificada del progreso. 
min, "Le surrealisme", apud Löwy, "Walter Benjamin y el surrealismo", 85), nos hace acordarnos del "realismo gótico"7 de Bajtín descrito en Rabelais en un registro extático e inspirado que presupone no simplemente un estilo, sino una concepción "cósmica" del cuerpo colectivo. ${ }^{8}$ El motivo utópico y mesiánico que acompaña la concepción de la historia en Benjamin puede encontrarse, de acuerdo con ciertas interpretaciones actuales de Bajtín (cfr., por ejemplo, Gardiner, "Bakhtin's Carnival"; Poole, "Bakhtin and Cassirer"), en toda la representación del carnaval como uno de los mecanismos de la historia, incluyendo la noción específica del lenguaje (de la plaza pública en particular). ${ }^{9}$ Desde luego, nos vemos obligados a trazar entre el "lenguaje puro" y el de la plaza pública (en el sentido que V. Turbín le atribuía) una analogía que no a todos gusta (desde el filósofo ruso A. F. Lósev hasta el analista contemporáneo B. Groys; cfr. Bubnova, En torno a la cultura, 150). Por lo pronto, nos vamos a limitar a esta analogía.

Uno de los descubrimientos más importantes de Bajtín es el crecimiento del sentido en el "gran tiempo". ${ }^{10}$ Esto quiere

${ }^{7}$ Término empleado en la fase de disertación de Rabelais. Cfr. N. Pan'kov, "Versión temprana de la concepción del carnaval".

${ }^{8}$ Es interesante apuntar que el "cuerpo colectivo" aparece también en Benjamin (cfr. el artículo "Walter Benjamin y el surrealismo"). Tal vez éste sea uno de los puntos en que las iluminaciones de los dos pensadores se corresponden con la cultura moderna. Este cuerpo colectivo puede encontrarse en Gogol —a quien Benjamin leyó- en la descripción del tumulto de una feria pueblerina, "cuando toda la muchedumbre se funde en un solo enorme monstruo y sacude todo su corpachón en la plaza y las angostas calles, grita, ríe, brama..." (Gogol, 16).

${ }^{9}$ Acerca del lenguaje de la plaza pública, ver V. Turbín, "Carnaval: religión, política, teosofía” [artículo del libro, en ruso, Bajtinski Sbornik I, Moscú, 1990, 6-29]. Turbín considera que el lenguaje de la plaza pública corresponde al júbilo con que el "Verbo encarnado", o sea Cristo, festeja el cuerpo con sus funciones y manifestaciones, para Él ajenas, en la misma medida en que el lenguaje terrenal se opone al lenguaje de "otros mundos", de donde el hijo de Dios viene (cfr. Bubnova, En torno a la cultura popular de la risa, 157 y 158).

${ }^{10} \mathrm{Cfr}$. Bocharov, 280. Me parece absolutamente fundamental la idea de Bajtín acerca del crecimiento del sentido en el tiempo. En el texto citado, S. G. Bocharov la plantea como entre comillas, teniendo posiblemente presente la crítica de M. L. 
decir que cada generación de lectores aporta su propia comprensión a los textos (no necesariamente literarios) conocidos desde siempre. Me pregunto si no es conveniente completar el concepto del "gran tiempo" con el del espacio, para acercarnos de esta manera al cronotopo en el "gran tiempo". ${ }^{11}$ La lectura de Bajtín después de una generación de investigadores cambiaba, como es bien sabido, con cada modificación de las condiciones de recepción. Simultáneamente iban surgiendo nuevos sentidos relacionados también con la difusión de sus ideas en el espacio, de modo que Bajtín está hace tiempo arraigado en Latinoamérica. Lo mismo se puede decir de Walter Benjamin, a quien colocamos al lado de Mijaíl Bajtín, convirtiendo esta operación en una variedad de lectura en el tiempo-espacio: ambos se han convertido en parte de un acervo intelectual universal, del que Latinoamérica reclama legítimamente su parte, al leer a los dos en una nueva perspectiva sobre el fondo de sus propios contextos culturales y políticos y de una nueva etapa del pensamiento. Tal situación se inscribe orgánicamente en la teoría del dialogismo bajtiniano:

Un texto vive tan sólo en contacto con otro texto (contexto). Sólo en el punto de este contacto de los textos se enciende una luz que ilumina hacia delante y hacia atrás, incluyendo el texto en un diálogo. Subrayamos que se trata del contacto dialógico entre los textos, y no de un contacto mecánico de oposiciones que únicamente es posible dentro de los límites de un solo texto (pero no del texto y los contextos) entre los elementos abstractos (signos dentro del texto), y que sólo es necesario en la primera

Gasparov (artículo de 1978, "Bajtín en la cultura rusa del siglo xx" [en ruso], 3336). Mientras tanto, en Benjamin se puede encontrar una idea afín en "La tarea del traductor".

${ }^{11}$ A. Gómez-Moriana propuso una diatopía como dimensión espacial del cronotopo (cfr. Gómez-Moriana, "Triple dimensionalidad del cronotopo bajtiniano...", 154). 
etapa (de la comprensión del significado, pero no del sentido) (Bajtín, Estética de la creación verbal, 364).

Por supuesto, se presenta el problema del estatus de la verdad y de la justificación de todas nuestras interpretaciones. No obstante, no hay que perder de vista que incluso un error o una lectura imprecisa generan en una situación determinada un desplazamiento del sentido e incluso un sentido nuevo, como consta a partir de los numerosos constructos "bajtinizantes" en las lenguas más diversas. ${ }^{12}$ Podríamos remitirnos a la "traducción total", estado que predomina en el mundo contemporáneo, ${ }^{13} \mathrm{el}$ modo de comunicación que presupone una transformación de las comunicaciones a través de los sistemas sígnicos variados, mismos que, a su vez, representan distintos niveles de diálogo. Sentidos nuevos coexisten e influyen sobre el pensamiento contemporáneo. La verdad, de acuerdo con Bajtín, también tiene un carácter dialógico o "de congregación" [соборньіü]. Así, en Voloshinov "la verdad sólo es eterna como generación permanente de la verdad" (El marxismo, 172). En Bajtín "la verdad requiere de una pluralidad de conciencias, [...] es por principio inabarcable por los límites de una sola conciencia, [...] al tener, por así decirlo, un carácter de acontecimiento, y nace en el punto de contacto de distintas conciencias" (Estética de la creación verbal, 92). En Benjamin, el contenido de la verdad se manifiesta mediante la alegoría concebida como una iluminación instantánea del sentido semejante a un relámpago,

${ }^{12}$ Comparto la opinión de Caryl Emerson acerca de que "translation, broadly conceived, was for him the essence of all human communication" (cfr. Bajtín, Problems of Dostoevsky's Poetics, "Editor's Preface", XXXI). Cfr. también Beasley-Murray, Mikhail Bakhtin and Walter Benjamin, 101: "Benjamin, like Bakhtin and Voloshinov, raises a secondary activity, translation, to the status of primary activity. Translation becomes the foundation for all language". Desde luego, el sentido que ambos pensadores atribuyen a la traducción no coincide: para Benjamin, en la traducción ha de manifestarse la palabra de Dios, mientras que Bajtín sugiere el crecimiento del sentido por medio de la traducción concebida como diálogo.

${ }^{13}$ Cfr. Torop, La traducción total, passim. 
así como mediante el lenguaje plural de las cosas (cfr. Jobim e Souza, "Mikhail Bakhtin e Walter Benjamín", 341).

Por otra parte, en el mismo sentido, Walter Benjamin nos invita a examinar más de cerca la transformación que opera la traducción en el original: por una parte, traduttore, traditore; por otra,

las traducciones que son algo más que comunicaciones surgen cuando una obra sobrevive y alcanza la época de su fama. Por consiguiente, las traducciones no son las que prestan un servicio a la obra, como pretenden los malos traductores, sino que más bien deben a la obra su existencia. La vida del original alcanza en ellas su exposición póstuma más que vasta y siempre renovada (Benjamin, Angelus Novus, 130).

Es decir, la traducción implica la emergencia de un nuevo sentido o — si se prefiere- la manifestación de un sentido hasta ahora oculto.

Por eso hablaremos de Bajtín y Benjamin desde nuestras circunstancias actuales, extrañas para ellos, a pesar de que en muchos aspectos esta lectura cruzada se inscribe en la perspectiva de las intuiciones o iluminaciones de los dos pensadores, a saber: desde el punto de vista del cronotopo contemporáneo, que presupone una recepción universal que atraviesa lenguas, territorios y condiciones sociales e intelectuales de un mundo en estado de cambio permanente e inusitadamente acelerado. La refracción de los sentidos resulta inevitable, incluso necesaria y debe ser analizada no sólo desde una verdad abstracta o de una adecuación teórica, sino desde el punto de vista de la vida social de la palabra.

Ambos pensadores son parte inalienable de aquel pensamiento filosófico contemporáneo que se concentra en torno al análisis de los destinos del siglo veinte y se inscribe en el contexto de la época postsecular que estamos viviendo. Tratamos de entender nuestro mundo, revisando la experiencia vivida en 
busca de elementos aptos para su evaluación y, por consiguiente, para orientarnos en la situación actual. Se trata, desde luego, de una nueva etapa de la búsqueda de la verdad, ya después de que las "grandes narrativas" del siglo pasado perdiesen su prestigio. ¿Acaso no es por eso que recurrimos a Bajtín y Benjamin? En realidad, la nuestra no es menos época de transición que aquella en que nuestros "héroes", actualmente convertidos en blanco de interpretaciones intrínsecamente contradictorias (y de contrapunto), empezaban a escribir: estamos en un nuevo "cambio de paradigma". No voy a demorarme en las bien conocidas etapas de la recepción del pensamiento bajtiniano (la escuela semiótica soviética, Kristeva, Todorov, Holquist, Morson-Emerson, la escuela postmarxista angloparlante - Gardiner, Shepherd, Brandist, Hirschkop y Hitchcock-, la tendencia teológica occidental y la inclusión de Bajtín en el ámbito de la Iglesia [votserkovlenie]) que en la década pasada presenciamos en la escuela de los intérpretes rusos; señalo sólo algunos momentos de dicha recepción durante los últimos cuarenta años. No está por demás mencionar también las particularidades de la lectura de Bajtín en el mundo hispanoparlante, señalando oportunamente las diferencias entre España y Latinoamérica. ${ }^{14}$ De este modo, la trayectoria del pensamiento bajtiniano con sus diversas interpretaciones transcurre desde la semiótica

${ }^{14}$ Mientras en España prevalece la recepción a la luz de la teoría y la historia literaria (cfr. Domingo Sánchez-Mesa), en América Latina se destaca un círculo más amplio de disciplinas de los proyectos ideológicos para los cuales la aportación de Bajtín resultó ser necesaria (basta revisar las actas de la "Bakhtin Conference" celebrada en Brasil en el año 2003). En México, las ideas de Bajtín llegaron a ser especialmente fructíferas para la antropología marcada por una determinada ideología (la misión histórica de la salvación de las culturas e identidades indígenas). Hay un cierto interés en el área de la historia del arte, de los estudios literarios y de la lingüística, disciplinas menos comprometidas ideológicamente. Cabe añadir, por otra parte, que en cierta medida en los Estados Unidos se observa una situación análoga en la antropología: la "antropología dialógica", basada en la conciencia del papel político tradicional de esta disciplina, impregnada de la ideología del colonialismo, surge bajo la influencia catalizadora de Bajtín. Cfr. Tedlock y Mannheim, eds., The Dialogic Emergence of Culture. 
y el marxismo hasta la teología: las exégesis de las ideas del pensador generan respuestas ideológicas, comenzando por la búsqueda de soluciones revolucionarias y terminando por su vinculación con la tradición rusa de filosofía religiosa.

A su vez, Walter Benjamín propone en sus textos una síntesis de la teología judía con el materialismo histórico concebido como análisis político de la cultura (cfr. Löwy, "Walter Benjamin y el surrealismo"). ${ }^{15}$ Para ejemplificar una interpretación no sintética de las ideas de Benjamin se puede confrontar la lectura contrastante del teólogo Gershom Scholem y del marxista Theodor Adorno. En Latinoamérica, uno de los promotores de la tendencia marxista es el filósofo Bolívar Echeverría. De este modo tenemos un misticismo judío confrontado al marxismo. Al mismo tiempo, Michael Löwy plantea una síntesis de estas tendencias contrastantes en la obra del pensador alemán. Por otro lado, las "iluminaciones" de Bajtín y de Benjamin se interpretan en América Latina en un sentido claramente político y sirven de fundamento para pensar los hechos de la historia y la cultura contemporánea e incluso para elaborar la metodología de diversas disciplinas, así como la estrategia de la posición u orientación política en la literatura y los estudios

${ }^{15}$ Para Löwy, las tres fuentes principales del pensamiento de Benjamin son el mesianismo judío, el romanticismo alemán y el materialismo histórico. Lo que se toma por la visión del mundo romántica puede ser definido como una crítica culturológica de la civilización contemporánea (capitalista) en nombre de los valores precapitalistas, crítica o protesta que se concentra en los aspectos más odiosos del capitalismo, a saber: cuantificación y mecanización de la vida, reificación de las relaciones sociales, descomposición de la comunidad y consiguiente decepción del mundo. Benjamin retoma la tradición del romanticismo revolucionario y su conjunto de ideas estéticas, teológicas e historiográficas. Mediante la tradición romántica entendida como cognición, arte y praxis, Benjamin ataca la ideología del progreso en nombre de la revolución. Esta característica formulada por Löwy debe modificarse a la luz de las divergencias manifiestas de Benjamin con respecto a los románticos en toda una serie de cuestiones teóricas y cosmovisionales, hecho que convierte su actitud hacia el romanticismo en claramente dialógica, lo cual recuerda las actitudes de Bajtín hacia toda una serie de antecesores suyos. 
literarios ${ }^{16}$ Existen modelos muy productivos de lecturas de las formas más actuales del arte (por ejemplo, nuevas teatralidades y performance concebidas como práctica política), formas cuyas realizaciones e interpretaciones se basan en determinadas lecturas de la antropología filosófica bajtiniana. ${ }^{17}$

Este no es, desde luego, el primer intento de colocar en una misma fila a Bajtín y Benjamin (cfr. como ejemplo Jobim e Souza, "Mikhail Bakhtin e Walter Benjamin"; Hirschkop, Mijail Bajtin: An Aesthetics y "O sagrado e o secular"; Tihanov, The Master and the Slave; Beasley-Murray, Mikhail Bakhtin and Walter Benjamin, entre otros). De acuerdo con BeasleyMurray, ${ }^{18}$ la misma inconmesurabilidad de ambos pensadores permite ver mejor a uno a la luz del otro, permitiendo distinguir en cada uno de ellos los aspectos invisibles capaces de manifestarse tan sólo en esta confrontación (cfr. Bajtín, Estética de la creación verbal, 335).${ }^{19}$ Esto hace recordar la idea de Bajtín acerca de la recíproca exotopía [outsideness] de las culturas en el proceso del contacto, gracias a la cual la posición de observador de una de ellas le permite distinguir en la otra aspectos inaccesibles a su propio autoanálisis. Por lo demás, conviene recordar la observación de S. S. Averintsev acerca del tema: ${ }^{20}$

${ }^{16}$ Cfr. Ortúzar, "Estéticas del residuo en Chile del postgolpe. Walter Benjamin y la escena avanzada", Acta Poetica, 28, 1-2, 2007, 111-127. Se trata de un ejemplo de cómo el pensamiento de Benjamin ayuda a organizar la experiencia colectiva de la superación de la dictadura en el contexto del arte contemporáneo. Existen investigaciones que se apoyan, con base en un material análogo y con los mismos propósitos, en la antropología filosófica de Bajtín. Cfr. Thorn, The Lived Horizon of My Being.

${ }^{17}$ Cfr. Diéguez, Escenarios liminales en Latinoamérica.

${ }^{18} \mathrm{Al}$ confrontar la filosofía del acto de Bajtín y las ideas análogas de Benjamin en torno al concepto de experiencia (Erfahrung y Erlebnis), este analista encuentra interesantes paralelos y describe la posición original de los dos pensadores en estética, ética y "filosofía de la vida" (ésta última no entendida en su acepción específica).

19 "Le planteamos a la cultura ajena nuevas preguntas, mismas que ella no se planteaba a sí misma, buscando en ella respuestas a estas preguntas nuestras, y la cultura ajena nos contesta y nos revela sus aspectos nuevos, sus nuevas profundidades de sentido".

${ }^{20}$ En el comentario para la defensa de la disertación doctoral de V. L. Makhlin. 
Debido a las circunstancias biográficas y cronológicas, comparar la creación filosófica de M. M. Bajtín con la de cualquier filósofo contemporáneo de Occidente, de la Rusia prerrevolucionaria o de la emigración rusa es lo mismo que comparar alguna tragedia perdida de la antigüedad clásica, de la que tenemos noticias gracias a algunos fragmentos, testimonios y en parte a sus reflejos en la literatura posterior, con una obra dramática de la Modernidad, que analizamos junto con las indicaciones autoriales para los actores (apud Makhlin, "Debemos hallar el camino", 149).

No obstante, no hemos de olvidar que incluso Aristóteles, antes de que se encontraran los originales griegos, fue primeramente conocido en Occidente por las traducciones árabes. Sólo podemos trabajar con lo que nos queda (ver los epígrafes al presente ensayo), pero conviene hacerlo con la reserva que nos sugiere la observación de S. G. Bocharov: Benjamin, a pesar de su marginalidad, ha sido mucho más difundido y comentado que Bajtín; ignoro si es mejor comprendido. Supongo que la comprensión es sólo posible en relación con nuestra propia época, y sólo cobra sentido a la luz de nuestra propia experiencia: "Llamo sentidos las respuestas a las preguntas. Lo que no contesta ninguna pregunta, para nosotros carece de sentido" (Bajtín, Estética de la creación verbal, 350). Es uno de los pensamientos de Bajtín que nos siguen inspirando y nos convierten en adeptos, con o sin razón.

Hace mucho ha sido reconocido que cuando Bajtín y Benjamin escriben sobre temas literarios, el resultado son textos llenos de tensión filosófica. Bajtín elaboró una concepción de la novela de educación y, en su base, la idea del cronotopo como modelo universal que da forma a la visión del mundo, en primer lugar en el ejemplo de Goethe, a pesar de que su perspectiva abarca obras literarias comenzando por la Antigüedad clásica $\mathrm{y}$, pasando por Rabelais, hasta la literatura de nuestro tiempo. $\mathrm{El}$ autor ruso retoma los parámetros kantianos de tiempo y es- 
pacio y los inscribe en la historia, relacionando con ellos las diferentes etapas de desarrollo de la sociedad. Tiempo y espacio son para él condiciones de posibilidad de la percepción humana del mundo en la historia, es decir, de la cosmovisión. Esto le permite, por una parte, elaborar una herramienta teórica para el análisis de las obras literarias y, por otra, mostrar de qué manera los conceptos historizados de tiempo y espacio condicionan la posición personalizada del sujeto cognoscente (cfr. Bajtín, Estética de la creación verbal, 371). De este modo inscribe esta teoría en una especie de "poética histórica" relacionando con ella su antropología filosófica, al revisar las relaciones sujetoobjeto en la Weltanschauung de Goethe. ${ }^{21}$

Tomaré como punto de partida la idea de Bajtín acerca del excedente de la visión del otro con respecto del yo, bastante productiva para comparar, tomando como ejemplo a la autoridad indiscutible de Goethe, los puntos de vista de los dos pensadores y los dos cortes culturales correspondientes, situados en planos contiguos. Y ahora pueden mirarse mutuamente, a través de la mirada mediadora del observador contemporáneo. Hace poco estos vecinos de la cronotopía - Bajtín y Benjamin- estaban separados por la cortina de hierro de los prejuicios ideológicos y por la distancia impuesta por las circunstancias políticas. Para ambos pensadores Goethe fue el pretexto y la inspiración para producir sus propias iluminaciones. Goethe está presente de una manera constante y activa en las investigaciones de Benjamin en torno a la literatura y la cultura, más allá del temprano ensayo sobre Las afinidades electivas. Es más, Benjamin está obsesionado con el genio de Goethe, que se le presenta en los sueños como si estuviera vivo. ${ }^{22}$ A su vez, para Bajtín, Goethe es uno de los tres principales "héroes" de su "autoría" aunque es difícil juzgar, por los textos que nos llegaron del pensador ruso, sobre el grado de su autoidentifica-

${ }^{21}$ Cfr. Bajtín, Estética de la creación verbal, 396 y 397.

${ }^{22}$ Cfr. Dirección única, cap. "No. 13". 
ción con el clásico alemán, como evidentemente le sucedió con Dostoievski. En cuanto al propio Goethe como personalidad, éste, como bien se sabe, se pensaba a sí mismo justamente en el "gran tiempo", como ofreciéndose a un diálogo suprahistórico: "Yo vivo en los milenios", solía decir (Eckermann 5.07.1827).

Es difícil negar que, con toda su inconmesurabilidad y contraposición, los dos pensadores son, a pesar de todo, como una especie de vasos comunicantes. Puesto que en la base de esta reflexión se encuentra Goethe, conviene ante todo recordar la fórmula de las "afinidades electivas" que está en el centro del famoso estudio benjaminiano de 1923, y que reaparece en los ensayos posteriores. A pesar de que Bajtín en sus investigaciones conservadas acerca de Goethe ni siquiera menciona la novela Las afinidades electivas, concentrándose en la "novela de educación", Poesía y verdad y Viaje a Italia, la novela en cuestión es sumamente "cronotópica" (como sus otras obras, o más) y por ende se presta a un análisis desde esta perspectiva teórica. Me permitiré un uso metafórico del concepto de las "afinidades electivas" para profundizar en el paralelo entre Benjamin y Bajtín. ${ }^{23}$ La metáfora de las "afinidades electivas" ya ha sido utilizada por el estudioso de la obra de Benjamin, M. Löwy (Rédemption et utopies), que ha definido en estos términos la correlación entre la teología judaica y el marxismo; además, el propio Benjamin la utiliza ampliamente en sus estudios sobre Baudelaire, por ejemplo (cfr. Benjamin, Obras II 1, 183, 185). A su vez, Löwy se apoya en un uso análogo de este concepto en Max Weber (La ética protestante, 1905). Es imposible reducir las relaciones dialécticas entre la teología y el materialismo dialéctico a una causalidad directa o a la influencia en el sentido tradicional. Según Löwy, se puede

\footnotetext{
${ }^{23}$ Recordaré que Goethe, siendo naturalista, elaboró la figura de las "afinidades electivas" a partir del concepto sacado de la obra del químico sueco Thorbern Olof Bergmann (1735-1784). Max Weber, por lo visto, toma prestada la idea tanto de las ciencias naturales del siglo XVIII como de Goethe.
} 
apreciar, en cambio, su analogía estructural, convergencia o atracción recíproca. Desde luego, al hablar de Bajtín y Benjamin en términos de las "afinidades electivas", no cabe proponer una fusión sino que más bien se trataría de las relaciones dialógicas accesibles tan sólo a la mirada de un investigador interesado. Se trata justamente de las "afinidades electivas": sus destinos hasta cierto punto son análogos sin coincidir totalmente (como su marginalidad); sus puntos de partida intelectuales, el círculo de sus intereses, sus fuentes, en parte coinciden (Kant, el neokantismo, la presencia del marxismo), pero en ocasiones son opuestas. Ahora bien, los observamos a los dos desde nuestro tiempo, recurriendo a estos autores por razones análogas. Por eso, cuando Bajtín describe el camino de la palabra hacia su objeto, recordando las muchas "voces" que acompañan y cuestionan cada uno de los sentidos que es susceptible de adoptar la palabra, ${ }^{24}$ conviene recordar las siguientes palabras de Benjamin:

[...] Il ne s'agit pas de présenter les oeuvres littéraires dans le contexte de leur temps, mais bien donner à voir dans le temps qui les connaît -c'est-à-dire le nôtre. La littérature devient de la sorte un organon de l'histoire, et lui donner cette place - au lieu de faire de l'écrit un simple matériau pour l'historiographie-, telle est la tâche de l'histoire littéraire ("Histoire littéraire et science de la littérature", 283).

Esta visión de la literatura como un organón de la historia es mucho más cercana a la perspectiva dialógica y cronológica de Bajtín que al inmanentismo estricto que caracteriza al ensayo de Benjamin sobre Las afinidades electivas (publicado en 1924-1925). Benjamin escribe este último trabajo en la tradición de la filosofía romántica, de acuerdo con el concepto de

\footnotetext{
${ }^{24}$ Cfr. Bajtín, "Formas del tiempo", 90 y 91 sobre la manera en que "la palabra concibe su objeto".
} 
crítica literaria que los románticos alemanes tenían, ${ }^{25}$ tema al que dedica su famosa disertación. ${ }^{26}$ Se trata de la recuperación de una tradición crítica en la cual un texto crítico aspira a igualar su valor con el del objeto al que va dirigido. En este sentido se puede apreciar la ambición del entonces joven Benjamin, quien pretende medir fuerzas con el propio Goethe: un impulso plenamente romántico. (Quisiera recordar que desde el estudio de Todorov de 1980 se suele inscribir la obra de Bajtín en la tradición romántica. Recientemente, Bénédicte Vauthier ha planteado este complejo problema mostrando en qué forma el sentido de los textos de Bajtín se refracta en el discurso crítico condicionado de Todorov) ${ }^{27}$ Es necesario agregar que en la mencionada disertación, Benjamin ocupa una postura más distante con respecto al romanticismo que en el ensayo acerca de Las afinidades electivas, donde parece poner a prueba la densidad filosófica y las posibilidades estilísticas del punto de vista romántico. Así pues, su concepción se deslinda de la estética de lo sublime, que se remonta a los románticos, misma que coloca al arte en el lugar de una nueva religión de la época secularizada. Benjamin más bien hace eco de su teoría lingüística diseñada ya hacia $1916,{ }^{28}$ en la cual critica la idea

25 "La méthodologie utilisée dans l'étude sur le Trauerspiel trouve sa première expression dans le premier chapitre de l'essai sur Les affinités électives, où l'on voit aussi se déployer la position critique que Benjamin adoptera toujours à l'égard du mythe" (Palmier, Walter Benjamin. Le Chiffonier, 380).

${ }^{26}$ El concepto de crítica de arte en el Romanticismo alemán, Rolf Tiedemann y Hermann Schweppenhäuser (ed.), trad. Alfredo Brotons Muñoz, en Obras I 1: "Las teorías estéticas de los primeros románticos y de Goethe se basan en principios opuestos" (109).

${ }^{27}$ Vauthier, "Bakhtine, lecteur de Goethe". Lo que por lo pronto queda claro es que la presencia o ausencia de los conceptos románticos o, digamos, de las categorías kantianas, tanto en Bajtín como en Benjamin, debe ante todo apreciarse desde posiciones dialógicas, fructíferas, pero a menudo subversivas, y no como una tradición directa o resultado de una "influencia".

${ }^{28}$ Como Benjamin, los románticos transformaron hasta tal punto los propósitos de la crítica que una obra de pensamiento crítico se volvía indistinguible de las obras de arte. Pero al eliminar la diferencia entre el texto crítico y su objeto, des- 
de la arbitrariedad del signo que predominaba en la lingüística contemporánea, apelando a la magia de la palabra divina. Con base en esta concepción del lenguaje articula la crítica de la obra mencionada de Goethe. Su visión del arte presupone una postura ética basada en nociones teológicas, notablemente diferentes a las del romanticismo (la crítica del papel del genio, por ejemplo), a pesar de cierta coincidencia en la orientación formal. Cabe hablar de una actitud dialógica hacia la teoría del arte de los románticos, misma que se proyecta hasta la época del propio Benjamin en la obra de Gundolf, cuyo nombre evidentemente está asimismo presente en el contexto de las investigaciones bajtinianas acerca de Goethe, si partimos del análisis de sus fuentes. ${ }^{29}$ De acuerdo con Benjamin, la teoría del arte concebida con base en una dialéctica entre el orden divino y el humano, se contrapone a la teoría del arte contemporáneo que se puede encontrar en Stefan George y Gundolf (Weigel, "La obra de arte como fractura", 187-188). ${ }^{30}$ Por su parte, Baj-

aparecía asimismo la aspiración a una comprensión racionalizada. De esta manera, los románticos dejaron de lado el intento de concebir la crítica como la formación del pensamiento, en tanto modo mediante el cual el pensamiento y la comprensión pueden ser representados (cfr. Ferris, Cambridge Companion, 11). Pero en el trabajo sobre Las afinidades electivas, sin rechazar las tareas del pensamiento crítico (de la búsqueda del "contenido de verdad"), Benjamin se acerca al ideal estético de los románticos en el sentido del valor intrínseco del texto crítico.

${ }^{29}$ A pesar de la seguridad con que Tihanov (The Master and the Slave) señala a Gundolf como fuente de Bajtín (en relación con el problema de la visibilidad del tiempo, por ejemplo), no me queda del todo claro hasta dónde esto pueda ser cierto, porque en Bajtín es difícil encontrar el mismo espíritu con el que está impregnada la investigación de Gundolf. ¿Cuándo exactamente pudo Bajtín tener acceso al libro del filólogo alemán acerca de Goethe? ¿No lo habrá conocido en la edición de 1922, hacia 1940, mientras que S. S. Averintsev y S. G. Bocharov fechan la redacción del texto sobre la novela de educación en 1936-1938? (cfr. Bajtín, Estética de la creación verbal, 395$)$.

${ }^{30}$ Bajtín se acerca a las obras de Goethe desde un "realismo" concebido como valor intrínseco, que se afirma en la idea de una generación incesante: el ser humano en el proceso de generación en el marco de un permanente desarrollo del mundo histórico. Esta concepción parece bastante alejada de la exaltación postromántica del genio de Goethe como representante del "espíritu alemán” que puede encontrarse 
tín analiza la obra y las ideas de Goethe tomando en cuenta la autoapreciación del clásico alemán, es decir, desde la posición que se suele definir como "clásica" y por principio opuesta a la estética individualista de los románticos:

Se habla mucho de la originalidad, pero ¿qué exactamente ésta significa? Al nacer, el mundo empieza a influirnos, y así sigue hasta el final de nuestra vida. ¿Qué podemos nombrar como nuestro propio, aparte de la energía, la fuerza, el deseo? Si yo pudiera tan sólo señalar todo aquello que debo a mis grandes antecesores y contemporáneos, al excluir todo aquello hubiese quedado muy poco (Eckermann 12.05.1825).

En relación con este tipo de opiniones de Goethe, Bénédicte Vauthier ("Bakhtine, lecteur de Goethe") observa acertadamente: "Es inútil insistir largamente en el hecho de que podemos no solamente oponer estas ideas a la estética romántica, sino que también -y sobre todo — iencontrarlas en el primer plano en Bajtín!"31

Benjamin, como es sabido, en los años veinte dedicó a Goethe otro ensayo, destinado para la publicación en la enciclopedia soviética. Este texto está escrito con orientación al materialismo histórico; no sólo su tema era de interés en aquel periodo, sino uno de los fundamentos de su visión del mundo (en combinación, como se ha señalado antes, con la teología). Claro, su

en Gundolf, que estaba cercano, como es sabido, al círculo de Stefan George. "Fr. Gundolf convierte a Goethe en el portavoz de su propio 'yo', en un 'genio fuera del tiempo', lo examina como una imagen autónoma, cerrada en sí misma y carente de relaciones externas y de propósitos. Goethe, en su opinión, poseía una naturaleza antisocial congénita, y antes de que el ser humano empiece a tener vivencias, debe ser, y este ser es un protofenómeno irresoluble. A Gundolf no le interesa en absoluto el Goethe histórico, para él sólo existe un Goethe en cuanto mito, personificación del espíritu alemán" (firmado por F. Schiller [texto en ruso]; cfr. <www.magister.msk. ru/library/personal/shilf001.htm>).

31 "Inutile d'insister longuement sur le fait que nous pouvons non seulement opposer ces idées à l'esthétique romantique, mais aussi — et surtout— les retrouver au premier plan chez Bakhtine!" (Vauthier, "Bakhtine, lecteur de Goethe” [en prensa]). 
materialismo histórico figura en una forma bastante transformada y no comprometida con respecto a la versión soviética. En este artículo algunos párrafos están dedicados a Las afinidades electivas. Benjamin analiza aquí principalmente los problemas éticos y las relaciones familiares entre los personajes en el contexto de un feudalismo en proceso de extinción. Esta visión de la novela contrasta con la interpretación simbólica y mitológica del mismo conflicto en el trabajo anterior. Parecería que en el artículo enciclopédico Benjamin pone a prueba, más que un estilo, un punto de vista. ¿Acaso no recuerda este ejercicio las definiciones bajtinianas de la estilización en primer lugar y, en parte, las de la parodia, sólo en un nivel muy profundo, es decir, con cierta dosis de convicción o de autosugestión y, tal vez, de ironía dirigida a sí mismo?

Si analizamos los textos de Goethe desde el punto de vista del cronotopo bajtiniano, justo en Las afinidades electivas el novelista es especialmente cronotópico, pero hay que reconocerlo: la novela parece contener más de un solo cronotopo. Una visión materialista de la naturaleza y del paisaje predomina en la parte inicial de la novela, donde las huellas de la acción humana y del hombre hacedor se encuentran en el centro de atención del autor, ${ }^{32}$ pero luego el cronotopo en que actúa el homo faber se transforma, conforme se desarrolla el argumento, en uno "romántico": se presenta un paisaje romántico que tiende a una inmóvil eternidad. La cualidad de la mirada autorial cambia de tal manera que la naturaleza se animiza, adquiere cualidades antropomorfas y corresponde al estado de ánimo de los héroes. El carácter simbólico de estos elementos fue invariablemente señalado por la crítica, aunque según Benjamin se trata más bien de una alegoría. En lo que toca a los héroes, al menos dos de ellos - Ottilie y Edouard - se van convirtiendo en figuras míticas de los eternos enamorados a los que no les

${ }^{32}$ Aunque Benjamin señala, en el artículo enciclopédico, el carácter improductivo y decorativo de esta actividad. 
es dado unirse en este mundo, ${ }^{33}$ y se supone que se van a reencontrar en el más allá. La atracción basada en las "afinidades electivas" se presenta en medio de un conflicto ético y ya no corresponde al paradigma científico a partir del cual el autor empezó a introducir este motivo. Es posible que por esta razón Benjamin desarrolle su ensayo sobre la novela en una clave estilística afín a la tradición romántica, a la que Goethe en cuanto teórico se oponía. El propio Benjamin cuestiona esta tradición en su disertación doctoral: se da cuenta de que las "teorías estéticas de los primeros románticos y de Goethe se basan en los principios opuestos" (Benjamin, Obras II 1, 109). De acuerdo con Goethe, una crítica metódica, es decir, concentrada en su objeto, no se justifica. Y por el contrario, por más paradójico que resulte, desde el punto de vista de la concepción romántica del arte, un texto crítico puede ser equivalente o incluso superar el valor estético de su objeto. Al mismo tiempo, Benjamin busca encontrar mediante su crítica un "contenido de verdad", es decir, una verdad filosófica o incluso teológica, dejando el trabajo del comentario (el "contenido objetivo") para los filólogos, "técnicos" del análisis literario.

Ahora bien, la "estética del ojo" constituye la parte fundamental del cronotopo "realista", sobre la cual se construye en Bajtín la investigación sobre los textos de Goethe. A su vez Benjamin, fino analítico de la vista en una época ulterior de su trayectoria creadora (cfr. "Charles Baudelaire como lírico de la época del capitalismo maduro", 1938), en el ensayo sobre Las afinidades electivas todavía no le presta a la estética del ojo la misma atención que le merecería en el análisis de un París del Segundo Imperio. ${ }^{34}$ En el trabajo de 1938, dedica-

${ }^{33}$ A pesar de la opinión extratextual del propio Goethe en el sentido de que Edouard, en cuanto persona, no le gusta (cfr. Eckermann); aún así se deja llevar por la dinámica de su discurso. Esto recuerda las sugerencias de Bajtín acerca de la autonomía de los personajes de Dostoievski.

${ }^{34}$ Cfr. "París de la época del Segundo Imperio en la obra de Baudelaire" (Benjamin, Obras II 1, 150 y 151). 
do al momento histórico perteneciente a otra formación social, momento totalmente ajeno a la experiencia de Goethe (quien, como señala Benjamin en el artículo enciclopédico, la mayor parte de su vida no abandonó Weimar y evitaba con todas sus fuerzas visitar ciudades grandes), el crítico alemán muestra que la confianza en la vista como instrumento de la cognición desde luego se subvierte. Además, el paisaje citadino a la luz del cual se analiza la visión de Baudelaire no favorece un estudio naturalista en cuyo contexto se mueve el pensamiento de Goethe analizado por Bajtín. ${ }^{35}$ Una situación análoga puede encontrarse en Gógol, cuyo sentido de vista material Benjamin aprecia positivamente (en "La feria de Sorochintsy"). Pero cuando la mirada de Gógol se traslada hacia las calles de San Petersburgo, pierde inmediatamente la claridad y definición de la percepción, descubriendo lo engañoso de las apariencias. (Recordemos " $\mathrm{La}$ avenida Nevsky": "Miente a toda hora esa avenida Nevsky, pero más que nunca cuando la noche la oprime con su espesa masa..., cuando el mismo demonio enciende las lámparas sólo para mostrar todo en su aspecto inauténtico".) Este cuadro sí que es perfectamente comparable con los del París baudelairiano, a pesar de que desconozcamos si Benjamin habrá acompañado a Gógol en sus paseos literarios por San Petersburgo (es decir, si leyó o no Las narraciones petersburguenses), como lo hizo con los textos del cantor de la capital francesa en los tiempos de Napoleón III.

En cuanto a la concepción bajtiniana del lenguaje, ésta, como es sabido, se opone bastante al misticismo expiatorio de Benjamin, justamente en la medida en que Bajtín comparte los principios filosófico-lingüísticos de su círculo (no entraré aquí en la polémica acerca de la autoría de los conocidos trabajos en disputa firmados por Voloshinov, Medvédev, Kanaev). No obstante, me gustaría señalar que en los trabajos firmados por

\footnotetext{
${ }^{35}$ Cfr. Tihanov, The Master and the Slave, 237.
} 
el propio Bajtín se pone de manifiesto la simultaneidad del contexto social inmediato del enunciado con respecto al nivel ontológico de la comunicación, que nos remite permanentemente al concepto del "gran tiempo". En particular esto se detecta en la misma concepción del "supradestinatario" o del "tercero", mismo que puede ser representado por el pueblo, o las generaciones futuras, o por el propio Dios. Es necesario subrayar la co-presencia de los niveles cotidiano y ontológico en la concepción de un ser "único y unitario" ${ }^{36}$ de tal modo que el "acontecimiento" del acto y de la comunicación situado dentro de este ser está arraigado en el tiempo y espacio concretos, pero está determinado por una responsabilidad a la vez concreta y ontológica, supra-existencial. Es justamente lo que distingue el pensamiento filosófico-lingüístico de Bajtín en comparación con los textos "deuterocanónicos". ${ }^{37}$ En Problemas de la poética de Dostoievski (1963) la misma concepción del diálogo "en la eternidad" hace contrapunto con una concepción análoga de la comunicación lingüística en Benjamin. Lo que en realidad diferencia a los dos pensadores es la omnipresencia de la discursividad ideológica en la misma estructura de la conciencia y en general el papel que la discursividad desempeña en la antropología filosófica del ruso, ${ }^{38}$ mientras que para el alemán el

36 "El prosaísmo de Bajtín es un prosaísmo aparente" (Bonetskaia, "Observaciones sobre un fragmento del Autor y héroe", 255). Por prosaísmo se entiende la interpretación global de la obra de Bajtín como prosaica (en contraposición a la poética), a saber: en el plano de una filosofía de lo cotidiano, propuesta por Morson y Emerson en su libro de 1990 (Creation of a Prosaics). Mientras tanto, esta simultaneidad del nivel cotidiano y del ontológico fue señalada también por Holquist (en "Why Is God's Name a Pun?” y Dialogism (New Accents), 24). Cfr. la discusión del mismo problema por L. A. Gogotishvili (en Bajtín, Sobranie Sochinenii 5, 396-398).

${ }^{37}$ Agradezco el señalamiento que me hizo Serghei Sandler en el sentido de que la idea de la "fuerza mesiánica débil" (en Las tesis de la filosofía de la historia) tal vez ponga de manifiesto una intuición de Benjamin, paralela a la de Bajtín, acerca de la simultaneidad de los planos cotidiano y ontológico de la vida.

${ }^{38}$ Algunas coincidencias interesantes entre los dos pensadores pueden encontrarse al confrontar el ensayo benjaminiano sobre el lenguaje de 1916 con los apuntes bajtinianos de los años cuarenta. Bajtín habla del acto fundacional de la nominación 
"lenguaje de la verdad" no es el de la comunicación cotidiana y social, sino la plena autoexpresividad del ser. Benjamin hace resurgir en la memoria el lenguaje de las cosas, el lenguaje de un mundo anterior a la Caída, es decir, un lenguaje que transmite los contenidos espirituales. Este lenguaje expresa el ser espiritual que no puede ser transmitido mediante el lenguaje, sino tan sólo en el lenguaje. El ser espiritual es idéntico al ser del lenguaje tan sólo "en la medida en que puede ser transmitido. El ser lingüístico del hombre consiste en que el hombre da nombre a las cosas" (cfr. Benjamin, Obras II 1, 146-147). Mediante el nombre, el ser espiritual del hombre se comunica con Dios.

El Janus bifronte —imagen que aparece también en Benjamin- personifica la co-presencia de los planos ontológico y vital que caracteriza el pensamiento de Bajtín. Esta ruptura con el "teoretismo abstracto y fatal" que hace estos dos planos herméticamente incomunicables se manifiesta en muchos niveles. Se puede hablar aquí de una "relación dialógica", pero también de una "inversión carnavalesca".

La cuestión de las "fuentes" de ambos pensadores debe, desde mi punto de vista, replantearse radicalmente en una clave dialógica. En Bajtín, por ejemplo, encontramos una actitud irreverente hacia los conceptos de Kant, por ejemplo. Bajtín transforma la arquitectónica sistemática del filósofo alemán en una arquitectónica de las relaciones interpersonales perteneciente a la filosofía moral concebida incluso como una prima philosophia. Su descripción de la realidad ética del acto, que

que realiza el hombre inspirado por Dios, acto mediante el cual el lenguaje del mundo y de sus objetos se pone a funcionar. Bajtín, como ya sabemos, habla del "ser expresivo y hablante". Las reflexiones de Bajtín acerca de la trascendencia del nombre y del apodo, así como su evocación marginal de la doctrina mística de A. F. Losev acerca de la "glorificación del nombre", u onomatodoxia (имяславие), cercana por su espíritu y por sus fuentes a la concepción lingüística de Benjamin, van en una dirección análoga. 
se remonta supuestamente, de acuerdo con su terminología, al neokantismo, en realidad se transforma también dialógicamente "rebasando los límites del sistema de Cohen" (cfr. Nikolaev, "La escuela filosófica de Nevel”, 33). En realidad, no es sino la transformación del pensamiento ajeno que se lleva a cabo para que éste permanezca vivo: el modo más normal de la supervivencia de los conceptos en la historia del pensamiento filosófico, aunque en Bajtín este procedimiento deriva en la elaboración de la forma estéticamente acabada de una filosofía dialógica.

A su vez, Benjamin elabora su concepción de la experiencia a partir de las categorías de Kant, para transformar Erfahrung en una categoría universal que garantiza la transmisión discursiva responsable del contenido de la experiencia ("El narrador"), neutralizando la fugacidad y el carácter singular y particular de Erlebnis. De este modo se deslinda de la "filosofía de la vida", pero asimismo de Kant (Benjamin, Obras II 1, 172), ${ }^{38}$ al relacionar la cognición con el lenguaje y, a través del lenguaje, con la experiencia.

El motivo profundo de mi propuesta tal vez no sea demasiado manifiesto sobre el fondo general de una confrontación, por lo pronto superficial, de estas dos figuras que se adueñaron de la reflexión de toda una generación de profesores universitarios a nivel mundial, figuras señeras que desgraciadamente están pasando de moda. Lo que se pretende aquí también es hacer un llamado a una determinada autocrítica, que debería alentar, por fin, un análisis del papel de nuestra crítica universitaria en esta historia donde nuestros ídolos de ayer ahora están desbancados. "Esclavo" (aunque sea hegeliano), "Caín" e incluso

${ }^{38}$ Benjamin muestra que la cognición, a pesar de su carácter a priori e invariable, comparable a las matemáticas, no se expresa mediante fórmulas ni números, sino mediante el lenguaje, y que Kant no había logrado integrar por esta razón determinados aspectos de la cognición, tales como la religión, su forma suprema, en su sistema (“Sobre el programa de una filosofía futura", 1918). 
Ser Ciappelletto, el héroe de Boccaccio (Decameron, I, 1). En efecto, la historia de aquel gran pecador que en el lecho de muerte se convirtió en santo, sólo para salvar de la deshonra a aquellos que lo acogieron, recuerda bastante la historia de la recepción del pensamiento bajtiniano, y no sólo en Occidente. En el proceso de su confesión, Ser Ciappelletto permite al monje interpretar sus palabras en el sentido conveniente al caso, y los comerciantes "virtuosos" que lo hospedaron lo oyen y permiten la broma que, después de la muerte del pecador, tiene un efecto inesperado: sus restos se convierten en reliquias sagradas $y$, verdaderamente, hacen milagros. Hace mucho fue señalado que las ideas de Bajtín poseen para un investigador un carácter heurístico, desempeñando el papel de catalizador del pensamiento propio, y no somos quién para emitir juicios acerca de las circunstancias que las generaron: "no hemos estado ahî", parafraseando la expresión de Ajmátova ("нас там не стояло"). Pero todos nosotros, mercaderes interesados, participamos en el proceso en que "todo está a la venta" (recordando la película de Wajda). ${ }^{39}$

Hemos de terminar con un llamamiento - bastante radical— de Benjamin dirigido a los críticos: " $Q$ Quién no es capaz de tomar partido, que se calle!". ${ }^{40}$

${ }^{39}$ Wszystko na sprzedaz, 1968 [Everything for Sale].

40 "Wer nicht Partei ergreifen kann, der hat zu schweigen" (Benjamin, Dirección única, 45). 


\section{REFERENCIAS}

Agamben, Giorgio, La potencia del pensamiento, trad. Flavio Costa y Edgardo Castro, Barcelona, Anagrama, 2008.

Bajtín, Mijaíl M., Problems of Dostoevsky's Poetics, Caryl Emerson y Wayne C. Booth (ed.), Minneapolis, University of Minnesota Press, 1984.

_- "Formas del tiempo y del cronotopo en la novela. Esbozos de una poética histórica", en Teoría y estética de la novela, Madrid, Taurus, 1989 [en ruso Voprosy literatury i estetiki, Moskva, Xudozhestvennaia Literatura, 1975, 234-407].

—_, Estética de la creación verbal, trad. T. Bubnova [1982], Buenos Aires, Siglo XXI, 2002.

—- Sobranie Sochinenii, t. 5, Moskva, Russkie Slovari, 1996; t. 2, Moskva, Russkie Slovari, 2002.

Beasley-Murray, Tim, Mikhail Bakhtin and Walter Benjamin. Experience and Form, New York, Palgrave MacMillan, 2007.

Benjamin, Walter, Angelus Novus, Barcelona, Edhasa, 1971.

—- Dos ensayos sobre Goethe, trad. Graciela Calderón y Griselda Mársico, Barcelona, Gedisa, 1996.

__, "El problema de la sociología del lenguaje", en Imaginación y sociedad. Iluminaciones I, trad. Jesús Aguirre, Madrid, Taurus, 1999, 157-194.

__ , "Histoire littéraire et science de la littérature", en Oeuvres, t. II, trad. del alemán, Maurice de Gandillac, Rainer Rochlitz y Pierre Rusch, Paris, Gallimard, 2000, 274-283.

—, Dirección única, Madrid, Alfaguara, 2002.

—, Ensayos escogidos, trad. H. A. Murena, México, Ediciones Coyoacán, 2006.

—, Obras, libro I, vol. 1, trad. José Navarro Pérez, Madrid, Abada Editores, 2007.

—, Obras, libro II, vol. 1, trad. José Navarro Pérez, Madrid, Abada Editores, 2007.

Bocharov, S., "El acontecimiento del ser" [en ruso], en M. M. Bajtin. Pro et contra, Moskva, RJGI, 2002.

Bonetskaia, N. K., "Observaciones sobre un fragmento del Autor y 
héroe”, en Bajtinología (investigaciones, traducciones, publicaciones) [en ruso], San Petersburgo, Altaya, 1995, 240-275.

Bubnova, Tatiana (ed.), En torno a la cultura popular de la risa, Barcelona, Anthropos, 2000.

Brandist, Craig, The Bakhtin Circle: Philosophy, Culture and Politics, London-Sterling [Vermont], Virginia, Pluto Press, 2002.

CASsirer, Ernst, "Goethe y la filosofía kantiana", en Rousseau, Kant,

Goethe, trad. Roberto R. Aramayo, México, Fondo de Cultura Económica, 2007, 233-280.

—_ Filosofía de la Ilustración, México, Fondo de Cultura Económica, 2008.

Cohen, Esther, "El mesianismo judío de Walter Benjamin", en Topografías de la modernidad. El pensamiento de Walter Benjamin, Dominik Finkelde et al. (ed.), México, Universidad Nacional Autónoma de México-Universidad Iberoamericana-Goethe-Institut Mexiko, 2007, 139-150.

DiÉGUEZ, Ileana, "Escenarios liminales en Latinoamérica. Prácticas escénicas y políticas", tesis de doctorado en letras, México, Universidad Nacional Autónoma de México, Facultad de Filosofía y Letras, 2006.

Eagleton, Terry, The Ideology of the Aesthetic, Oxford, Blackwell Publishers, 1998.

Echeverría, Bolívar, "Deambular: Walter Benjamin y la cotidianidad moderna", Litorales: Teoría, método y técnica en geografía y otras ciencias sociales, 5, 2004.

_- Sobre el concepto de la historia en Walter Benjamin, México, Era, 2005.

Eckermann, Johann Peter, Conversaciones con Goethe, trad. Cansinos Assens, en Johann Wolfgang von Goethe, Obras, vol. III, Madrid, Aguilar, 1985.

—, Razgovory s Goethe, trad. N. Jolodkovski, Moskva, Zajarov, 2003. FERris, David S. (ed.), The Cambridge Companion to Walter Benjamin, Cambridge, Cambridge University Press, 2008.

GARDINER, Michael, "Bakhtin's Carnival: Utopia as Critique", Utopian Studies, 3:2, 1992, 21-49.

Gasparov, M. L., "Bajtín en la cultura rusa del siglo xx" [en ruso], en V. V. A. A., M. M. Bajtín. Pro et contra, Moscú, RJGI, 2002, 33-36. 
GodzICH, Wlad, "Correcting Kant: Bakhtin and Intercultural Interactions", en Michael Gardiner (ed.), Mikhail Bakhtin, vol. II, London-Thousand Oaks-New Delhi, Sage Publications, 2002, 3-13.

Goethe, Johann Wolfgang von, Las afinidades electivas, trad. José María Valverde, Barcelona, Icaria, 1984.

__, Las afinidades electivas, trad. Manuel José González y Marisa Barreno, Madrid, Cátedra, 2005.

Gogol, Nicolás, Cuentos ucranios, León Mirlos (trad.), Buenos Aires, Espasa-Calpe, 1947.

Gogotishvili, L. y P. Gurevich (ed.), Baxtin kak filosof, Moskva, Nauka, 1992.

Gómez Moriana, Antonio, "Triple dimensionalidad del cronotopos bajtiniano: diacronía, diatopía, diastrofía”, Acta Poetica, 18-19, 1997-1998, 153-218.

Gurganus, Albert E., "Typologies of Repetition, Reflection, and Recurrence: Interpreting the Novella in Goethe's Wahlverwandtschaften", en Goethe Yearbook, XV, Simon Richter y Daniel Purdy (ed.), Rochester, Camden House, 2008, pp. 99-114.

Hirschkop, Ken, Mijail Bajtin: An Aesthetics for Democracy, Oxford, Clarendon Press, 1999.

_- "O sagrado e o secular: atitudes perante a linguagem em Bakhtin, Benjamin e Wittgenstein", trad. Carlos Alberto Faraco, en Vinte ensaios sobre Mikhail Bakhtin, Petrópolis, Editora Vozes, 2006, 146-160.

Holquist, Michael, "Why Is God's Name a Pun?", en The Novelness of Bakhtin, Jorgen Bruhn y Jan Lundquist (ed.), Copenhagen, Museum Tusculanum Press, 2001, 53-70.

—_, Dialogism (New Accents), Routledge, London, 2002.

Jobim E SouZA, Solange, "Mikhail Bakhtin e Walter Benjamin: polifonia, alegoria e o conceito de verdade no discurso da ciencia conmeporânea", en Beth Brait (org.), Bakhtin, dialogsimo e construção do sentido, Campinas [Brasil], Editora da Unicamp, 1997, 331-346.

Lósev, Alexei F., "Vesch i imia" [La cosa y el nombre (1930)], $<$ http://www.philosophy.ru/library/losef/name.html>. [Consulta: 6 de marzo de 2009.] 
Löwy, Michael, Rédemption et utopies. Le judaïsme libertaire en Europe centrale. Une étude d'affinité élective, Paris, Presses Universitaires de France, 1988.

_- Walter Benjamin: Avertissement d'incendie. Une lecture des thèses "Sur le concept d'histoire", Paris, Presses Universitaires de France, 2001.

_- "Walter Benjamin y el surrealismo: historia de un encantamiento revolucionario", Acta Poetica, 28: 1-2, 2007, 73-93.

MAKhlin, V. L., "Yo abandono la Revolución. Un programa" [artículo en ruso], en Bajtinski Sbornik III [Compilación Bajtiniana III], Moscú, 1997.

__, "Debemos hallar el camino de vuelta... transcripción de la disertación doctoral de V. L. Makhlin", Dialog. Karnaval. Jronotop, 2, 1998, 139-192.

Morson, Gary Saul y Caryl Emerson, Mikhail Bakhtin: Creation of a Prosaics, Stanford, Stanford University Press, 1990.

MAn, Paul de, "La tarea del traductor, de Walter Benjamin", trad. Juan José Utrilla, Acta Poetica, 9-10, 1989, 257-294.

Nikolaev, N. I., "La escuela filosófica de Nevel", en M. M. Bajtín y la cultura filosófica del siglo XX. Problemas de bajtinología [libro en ruso], San Petersburgo, 1991, 30-45.

Ortúzar Vergara, Macarena, "Estéticas del residuo en el Chile del postgolpe. Walter Benjamin y la escena de avanzada", Acta Poetica, 28: 1-2, 2007, 113-127.

PALMIER, Jean-Michel, Walter Benjamin. Le chiffonier, l'Ange el le Petit Bossu, Paris, Klincksieck, 2006.

PARIKov, N., "Versión temprana del concepto de carnaval" [en ruso], Voprosy Literatury, 5, $1997<\mathrm{http}$ ://magazines.russ.ru/voplit/1997/5/pankov/html>.

Poole, Brian, "Bakhtin and Cassirer: The Philosophical Origins of Bakhtin's Carnival Messianism", Bakhtin/"Bakhtin": Studies in the Archive and Beyond, The South Atlantic Quarterly 97: 3-4, 1998, 537-579.

SALMERÓN, Miguel, "La onerosa presencia: lectura de Las afinidades electivas por parte de Walter Benjamin", Paideia, 16, 1992.

Scholz, Bernhard F., "Bakhtin's Concept of 'Chronotope': The Kantian Connection", en David Shepherd (ed.), The Contexts of 
Bakhtin: Philosophy, Authorship, Aesthetics, Amsterdam, Harwood Academic Publishers, 1998, 141-172.

Simmel, Georg, Goethe. Kant y Goethe, Buenos Aires, 1949.

Tedlock y Mannheim (ed.), The Dialogic Emergence of Culture, Urbana-Chicago, The University of Illinois Press, 1995.

Thorn, Judith, The Lived Horizon of My Being. The Substantion of the Self \& Discourse of Resistance in Rigoberta Menchú, M. M. Bakhtin and Victor Montejo, Tempe [Arizona], Arizona State University, 1996 (Special Studies, 29).

Tinanov, Galin, The Master and the Slave: Lukács, Bakhtin, and the Ideas of Their Time, Oxford, Clarendon Press, 2000.

Torop, Peter, La traducción total [título en ruso Total'nyi perevod], Tartu [Estonia], Universidad de Tartu, 1995.

Turbin, V., "Carnaval: religión, política, teosofía" [artículo en ruso], en Bajtinski Sbornik I [Compilación bajtiniana I], Moscú, 1990, 6-29.

VAuthiER, Bénédicte, "Bakhtine, lecteur de Goethe ou des ambiguïtés de la réception française (Todorov, Genette, Schaeffer, Rastier) d'un soi-disant romantisme bakhtinien" [2005], en Die Romantik: ein Gründungsmythos der europäischen Moderne, Paul Geyer y Anja Ernst (ed.), Göttingen, Vaudenhoeck \& Ruprecht, 2008 (en prensa).

Voloshinov, V.N., El marxismo y la filosofía del lenguaje, trad. Tatiana Bubnova, Madrid, Alianza, 1993.

Weber, Max, La ética protestante y el espíritu del capitalismo, trad. Luis Legaz Lacambra, Madrid, Sarpe, 1984.

Weigel, Sigrid, "La obra de arte como fractura. En torno a la dialéctica del orden divino y humano en 'Las afinidades electivas de Goethe' de Walter Benjamin", Acta Poetica, 28: 1-2, 2007, 173-204.

Wohlfarth, Irving, "Sobre algunos motivos judíos en Benjamin", trad. Esther Cohen, Acta Poetica, 9-10, 1989, 155-205. 
